\title{
Early and sustained treatment modifies the phenotype of birdshot retinochoroiditis
}

\author{
Pascal B. Knecht • Marina Papadia • \\ Carl P. Herbort Jr.
}

Received: 10 September 2013/Accepted: 14 September 2013/Published online: 1 October 2013

(C) Springer Science+Business Media Dordrecht 2013

\begin{abstract}
In this single-centre retrospective case review, we investigate the long-term follow-up of birdshot retinochoroiditis (BRC) patients, analysing the impact of early, vigorous, and prolonged treatment on the evolution of indocyanine green angiography (ICGA) signs and fundus appearance. Treatment delay was calculated for each BRC patient, and patients were classified into two groups-treatment delay of $<10$ months (early-treatment group) and treatment delay of $>10$ months (delayed-treatment group). Fundus photographs and ICGA frames from the initial visit and from the last follow-up visit were assessed. Fundus photographs were evaluated for the presence of at least three circumpapillary, typical, rice-shaped birdshot lesions in one eye, inferior or nasal to the optic disc. ICGA pictures were evaluated for the
\end{abstract}

P. B. Knecht · C. P. Herbort Jr. ( $₫)$

Retinal and Inflammatory Eye Diseases, Centre for Ophthalmic Specialized Care (COS), Clinic Montchoisi, Rue de la Grotte 6, 1003 Lausanne, Switzerland e-mail: carl.herb@bluewin.ch

P. B. Knecht

Department of Ophthalmology, University Hospital

Zurich, Zurich, Switzerland

M. Papadia

Ospedale Antero Micone, Genoa, Italy

e-mail: marinapapadia@yahoo.com

C. P. Herbort Jr.

University of Lausanne, Lausanne, Switzerland presence of lesions (hypofluorescent dark dots, fuzziness). Differences were compared between the two groups and between the first visit and the last followup visit. In the early-treatment group, 5/6 patients had no characteristic BRC fundus lesions, but $7 / 7$ patients in the delayed-treatment group displayed typical lesions. At last follow-up, 5/6 early-treatment patients showed no fundus lesions, and 6/7 delayed-treatment patients retained their fundus lesions. At presentation, all 13 patients exhibited lesions on ICGA. At last follow-up, ICGA lesions had completely disappeared in 4/6 early-treatment patients and 3/7 delayedtreatment patients. Thus, early and sufficiently dosed inflammation-suppressive treatment can prevent the appearance of typical BRC fundus lesions. It is therefore crucial to perform ICGA to detect otherwise occult stromal choroiditis in suspected BRC cases and to initiate adequate therapy immediately.

Keywords Birdshot retinochoroiditis . Indocyanine green angiography $\cdot$ Rice-shaped depigmented fundus lesions · Early treatment . Early diagnosis $\cdot$ Birdshot phenotype

\section{Introduction}

Birdshot retinochoroiditis (BRC) is a rare bilateral retinochoroidal inflammatory disease without known systemic involvement that mostly appears in Caucasians. It was first described in 1980 by Ryan and 
Maumenee [1] and nearly concomitantly by Gass, who called the disease vitiliginous choroiditis [2]. The typical clinical appearance of the full-blown disease is characterised by low, often subclinical anterior segment inflammation, vitritis, retinal vasculitis involving large veins as well as small retinal capillaries [3], and rice-shaped hypopigmented choroidal lesions [4]. The presence of the HLA-A29 antigen is not only a very strong supporting finding for BRC diagnosis, but should probably be considered a diagnostic criterion because HLA-negative cases are extremely rare [5-9]. $\mathrm{BRC}$ is unique in producing dual independent inflammatory involvement of the choroid as well as of the retina, the latter being responsible for disease morbidity and functional impairment due to inflammatory damage [10, 11].

We previously showed that classical birdshot fundus lesions are not necessarily present at disease onset, although they are required for diagnosis by the published diagnostic research criteria for BRC (RCBRC) [12, 13]. BRC can be diagnosed during its subclinical stage when indocyanine green angiography (ICGA) indicates occult choroidal lesions, the presence of characteristic fluorescein angiography (FA) findings and positive HLA-A-29 testing. Waiting for the appearance of BRC fundus lesions leads to significant diagnostic delay [11]. The question arises whether this diagnostic delay affects the long-term course of BRC; the necessity of immediate therapy was not highlighted in older publications because BRC was considered to have little response to corticosteroids or immunosuppressives [1, 2, 14-16], but the main reason for under-treatment was the fact that visual acuity, rather than retinal function was used to make treatment decisions. Indeed, for too long the functional criterion for treatment was (erroneously) visual acuity and not visual field [17]. However, more recent reports provide evidence that the functional outcome of treated BRC patients is superior to nontreated patients, and studies attempting to determine the best possible treatment schemes have been conducted [18, 19]. Unfortunately, there is no universally accepted and applied treatment. Therefore, it is critical to assess the long-term follow-up of BRC patients in terms of various monitoring and treatment approaches.

We previously reported the beneficial use of ICGA for monitoring subclinical choroidal inflammation in the subacute and convalescent stages of
Vogt-Koyanagi-Harada (VKH) disease [20-22]. We and others demonstrated that even if no inflammation was clinically apparent in VKH disease, ICGA detected occult choroidal stromal lesions that indicated smouldering inflammation [23]. We and others assumed that this subclinical inflammation is the origin of sunset glow fundus (SGF), often described as part of the natural course of VKH disease [20,24,25]. We also showed that if inaugural $\mathrm{VKH}$ disease is treated early and for a sufficiently long period with sufficiently high doses of inflammation-suppressive therapy (IST), SGF can be avoided [20]. BRC is a stromal choroiditis like VKH disease, and hence it is reasonable to assume that similar management strategies might be applied to both diseases. BRC fundus lesions might be prevented in the same fashion that SGF can be avoided in VKH disease, as long as early, vigorous, and prolonged IST is given.

The aim of this study was to investigate the longterm follow-up of BRC patients in terms of the impact of early, vigorous, and prolonged IST on the evolution of ICGA signs and on the BRC phenotype in terms of its fundus appearance.

\section{Methods and patients}

This was a single-centre, retrospective case review of all patients with an ocular inflammatory disease seen at the Centre for Ophthalmic Specialized Care (COS), Lausanne, Switzerland, between 1995 and 2012. This study was performed in accordance with the Declaration of Helsinki.

All patients underwent a complete ophthalmological work-up routinely applied to patients with posterior uveitis. Routine examinations included Snellen bestcorrected visual acuity (BCVA) testing, slit-lamp examination, applanation tonometry, and funduscopy in mydriasis. In addition, at each major visit, a complete set of investigational procedures was performed that included laser flare photometry, computerised visual field testing, microperimetry (when available), optical coherence tomography (when available), fundus photography, and dual FA and ICGA.

Patients with the diagnosis of BRC were identified. As published earlier [11], our diagnostic criteria for BRC included vitritis and retinal vasculitis in either or both eyes, visual-field anomalies in either or both eyes, 
stromal choroiditis as evidenced by ICGA in both eyes, and the presence of the HLA-A29 antigen. An additional, but not obligatory, criterion was the presence of rice-shaped, depigmented 'birdshot lesions'. Patients with available initial and final follow-up fundus photography, ICGA, and a follow-up $>3$ years were included.

Treatment was proposed in cases with visual field disturbance. This treatment rarely consisted of subTenon's injections when unilateral functional deficit was present. In cases with bilateral decreased visual function (visual field or visual acuity), therapy was increased to dual systemic oral corticosteroids and immunosuppressants $(1-2, \pm$ biologicals $)$ with subsequent tapering of steroids over 4-6 months to no treatment or a dosage $<10 \mathrm{mg} /$ day.

Treatment delay was calculated for each patient, and patients were categorised into two groups - treatment delay of $<10$ months (early-treatment group) and treatment delay of $>10$ months (delayed-treatment group). Most patients in the latter group were treated late because of diagnostic delay and/or because they declined immediate treatment after diagnosis. Mean diagnostic delay and mean treatment delay were calculated for both groups.

Fundus photographs and ICGA frames from the initial visit and from the last follow-up visit (while the patient was still under immunosuppressive therapy) were assessed independently by two investigators (PBK and $\mathrm{CPH}$ ), who were blinded to the name of the patient. If there was disagreement, a third observer (MP) was asked to interpret the fundus photographs and ICGA pictures to reach a decision.

Fundus photographs were evaluated for the presence of at least three circumpapillary, typical, riceshaped birdshot lesions in one eye, inferior or nasal to the optic disc; these lesions were required for the diagnosis of BRC, particularly as suggested by the RCBRC. When lesions were present, fundus appearance was scored as mild ( $<6$ lesions; grade 1), moderate (6-10; grade 2), or severe ( $>10$ lesions; grade 3). Average fundus scores were compared in both groups. Patient phenotypes ('typical fundus lesions' meeting the RCBRC system or 'no fundus lesions') were recorded in both groups at presentation and at the last follow-up visit.

ICGA pictures were evaluated for the presence of hypofluorescent dark dots (HDDs) and fuzziness of choroidal vessels. ICGA scores were established according to the following grading scores $-<5$ HDDs in both eyes and no fuzziness of choroidal vessels corresponded to grade 0 (some hypofluorescent lesions in the absence of fuzziness were tolerated to eliminate hypofluorescence due to scars or to nonbirdshot lesions); 5-10 lesions bilaterally corresponded to grade 1 , which could be upgraded to grade 2 in cases with extensive fuzziness; 16-30 lesions bilaterally corresponded to grade 2 , which could be upgraded to grade 3 in cases with extensive fuzziness of the choroidal vessels or downgraded to grade 1 in the absence of significant fuzziness;

$>30$ lesions bilaterally corresponded to grade 3, which could be downgraded to grade 2 in the absence of significant fuzziness of the choroidal vessels. Average scores were compared in both groups.

The proportion of patients with BRC lesions and those without lesions at the last follow-up was compared in both groups using Fisher's exact test. Differences in mean diagnostic delay and mean treatment delay were compared between groups. Differences in the mean scores of BRC lesions and differences in mean ICGA scores were compared between groups and between the first visit and the last follow-up visit in both groups. Student's $t$ test was used for all of these statistical comparisons.

\section{Results}

\section{Demographics}

Out of 1,504 new patients with ocular inflammatory conditions who were seen between 1995 and 2012, 25 patients (1.66\%; 19 female, 6 male) were diagnosed with BRC; 13 of these patients had sufficient data, met the inclusion criteria and could be considered for the study (0.86 \%; 8 female, 5 male; Table 1$)$. All patients were Caucasian. The mean patient age at BRC onset was $50.4 \pm 7.7$ years. All patients tested positive for the HLA-A29 antigen.

Six patients had a treatment delay of $<10$ months since the first symptoms were recorded. The mean diagnostic delay for these patients was $5.83 \pm 2.7$ months and the mean treatment delay was $6.5 \pm 3.1$ months. Seven patients had a treatment delay of $>10$ months, with a mean diagnostic delay of $25.71 \pm 11.6$ months and a mean treatment delay of $51.14 \pm 32.1$ months. The length of follow-up after initiation of therapy did not 


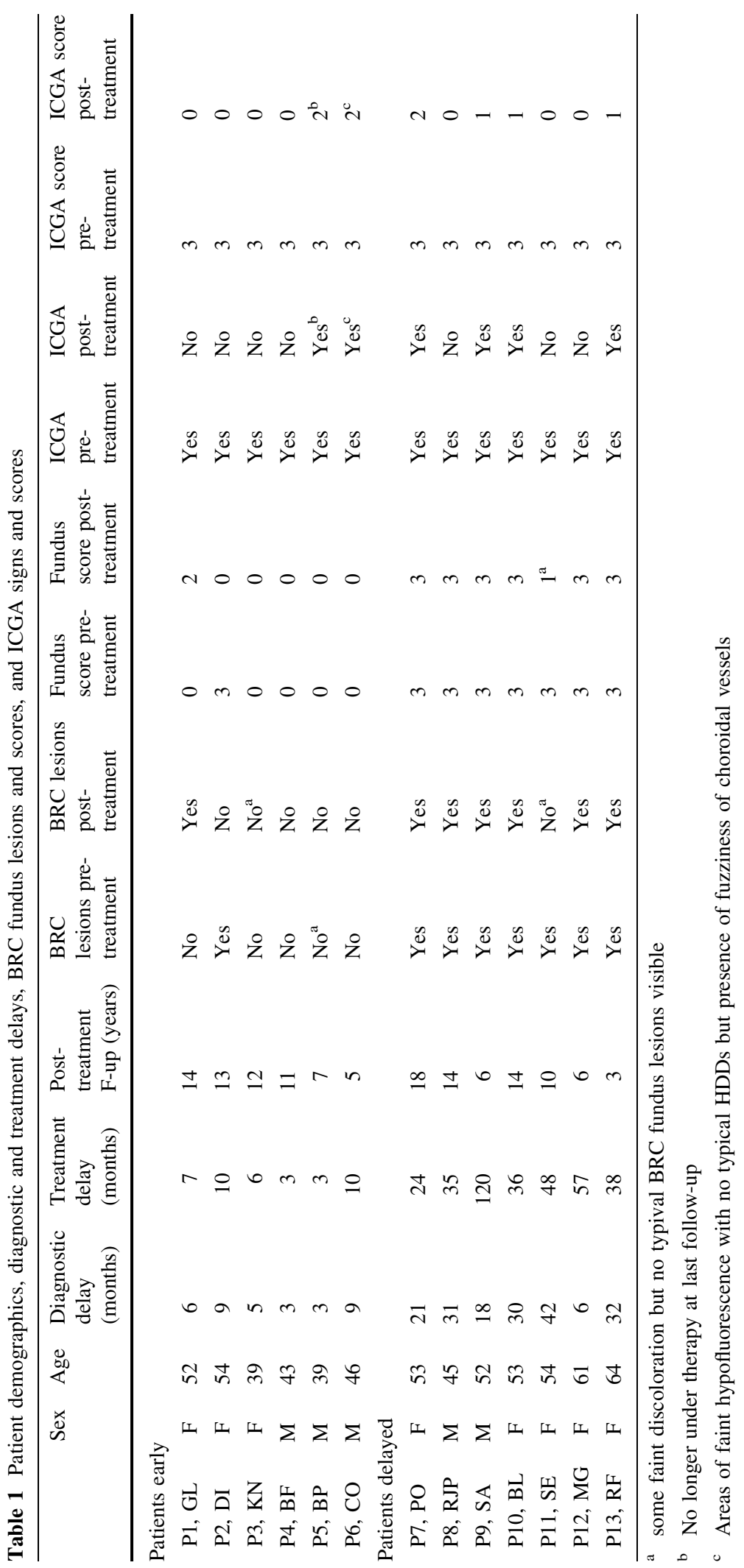


differ between groups $(10.3 \pm 3.6$ years in the earlytreatment group and $10.14 \pm 5.4$ years in the delayedtreatment group).

Out of 13 patients, only 8 displayed 'typical' birdshot lesions at presentation according to the RCBRC system. Five patients lacked fundus lesions; all of these patients belonged to the early diagnosis/ early treatment group.

\section{BRC fundus lesions}

At presentation, 5/6 patients in the early treatment group exhibited no characteristic BRC fundus lesions, with a fundus score of $0.5 \pm 1.2$. In contrast, 7/7 patients in the delayed-treatment group had typical BRC fundus lesions; all these lesions were scored as grade 3 and the mean fundus score of this group was $3 \pm 0$, which was significantly higher than the earlytreatment group $(p<0.0066)$.

At the last follow-up, 5/6 patients in the earlytreatment group had no fundus lesions. An illustrative case of such disease evolution is shown in Fig. 1. The lesions resolved and were no longer visible in one patient (Fig. 2), whereas another patient developed lesions despite treatment. In the early-treatment group, fundus score at the last follow-up remained low $(0.33 \pm 0.8)$ and was not significantly different from the pre-treatment score $(p=0.22)$.

At the last follow-up, 6/7 patients in the delayedtreatment group retained their fundus lesions, with a slightly lower fundus score of $2.71 \pm 0.7$ that was not significantly lower than the pre-treatment score of $3 \pm 0(p=0.17)$. This observation indicates that most lesions already had an irreversible component with permanent depigmentation that treatment did not affect (Fig. 3). In one patient, the BRC fundus lesions regressed, became faint, and no longer met the RCBRC criteria.

Taken together, these results demonstrate that only $1 / 6$ patients in the early-treatment group developed characteristic BRC fundus lesions at the end of followup; 5/6 patients never developed rice-shaped BRC fundus lesions. Six out of seven patients in the delayed treatment-group exhibited typical BRC fundus lesions at the end of follow-up. This difference was statistically significant (two-tailed $p<0.0292$ ) and was associated with a positive predictive value of 0.83 for not finding lesions with early treatment and a negative predictive value of 0.85 for finding lesions with delayed treatment (Fisher's exact test).

ICGA data

At presentation, all 13 patients displayed lesions (HDDs and fuzziness of the choroidal vessels) on ICGA, with a maximal grading of 3 for all patients (a mean score of 3 for the entire cohort and for both subgroups).

At the last follow-up, ICGA lesions had completely disappeared in $4 / 6$ patients in the early-treatment group, with a mean angiographic score of $0.66 \pm 1.03$, which was significantly lower than the score of $3.0 \pm 0$ before treatment $(p<0.0003)$. Similarly, ICGA lesions had disappeared in $3 / 7$ patients in the delayed-treatment group at the last follow-up; the mean angiographic score decreased to $0.71 \pm 0.7$, which was significantly lower than the score at presentation of $3.0 \pm 0(p<0.0007)$. Sometimes it was difficult to attribute HDDs to active lesions or to scars that were not indicative of activity. This difficulty pertains to all stromal choroiditis entities, as we previously described for VKH disease [26].

\section{Case illustrations}

Case 1 (patient 6, early-treatment group, no BRC fundus lesions before IST, no lesions after IST, some persistent ICGA activity; Fig. 1)

A male patient, aged 46 years at treatment onset, was diagnosed with BRC 9 months after the occurrence of visual disturbance consisting of a subjective decrease in visual acuity, dimness of vision, and floaters. Systemic IST was prescribed due to bilateral visual field defects that were noted on Octopus ${ }^{\circledR}$ perimetry (Octopus, Haag-Streit, Bern, Switzerland). At presentation, BCVA was 1.0 in both eyes and laser flare photometry indicated a subclinical aqueous flare of 7.0 $\mathrm{ph} / \mathrm{ms}$ in the right eye and $9.1 \mathrm{ph} / \mathrm{ms}$ in the left eye; there was a bilateral vitritis amounting to $2+$ cells (arbitrary slit-lamp score used in our institution), and the fundus was fuzzy but otherwise devoid of lesions (Fig. 1a, left sextet of images). Octopus ${ }^{\circledR}$ perimetry revealed a decreased mean defect as well as several focal scotomas. FA showed bilateral retinal vasculitis of the large veins and diffuse retinal hyperfluorescence due to capillary leakage, disc hyperfluorescence, and 


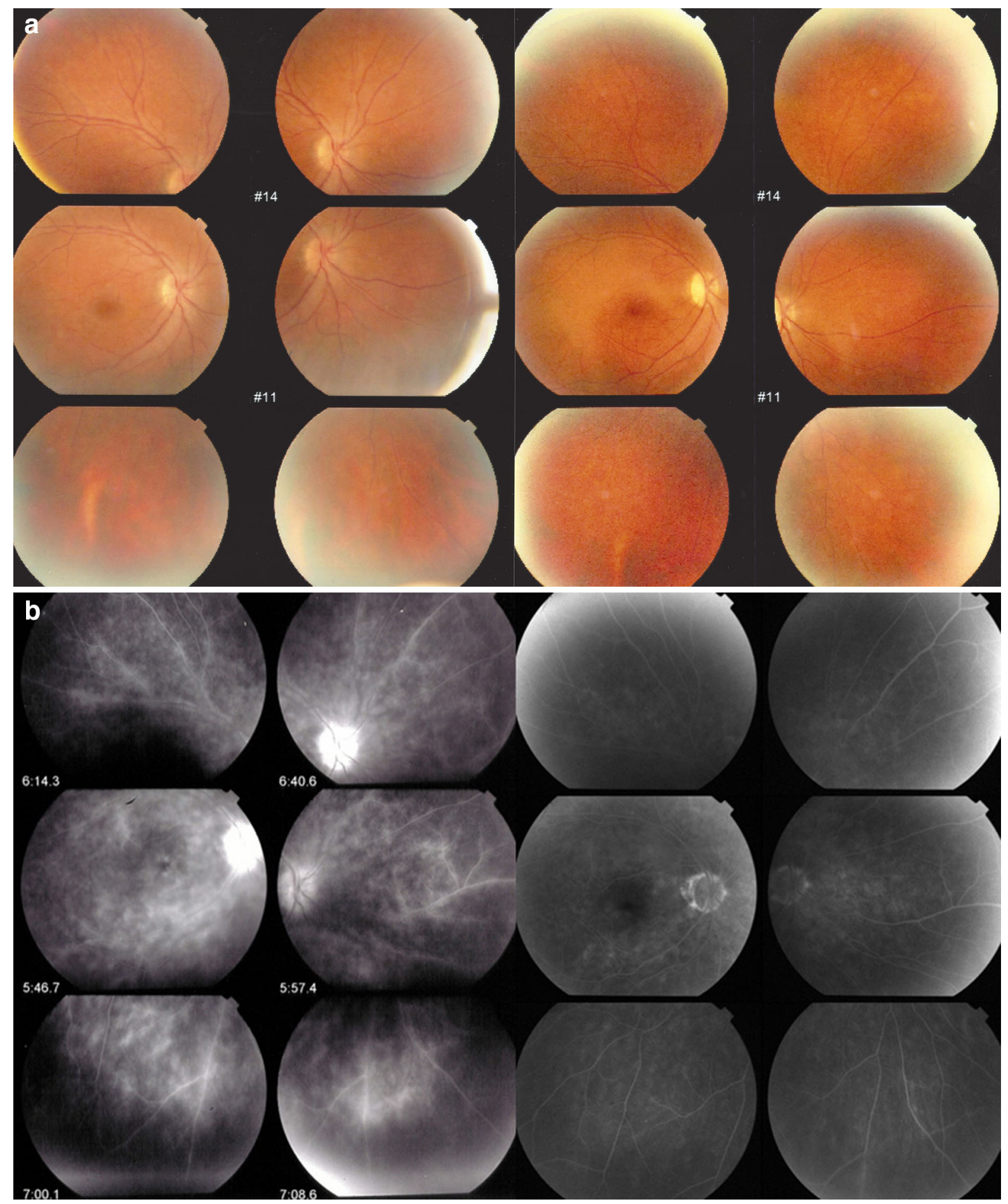

macular oedema (Fig. 1b, left sextet of images). ICGA indicated numerous HDDs as well as pronounced fuzziness of the choroidal vessels, yielding an angiographic score of 3 (Fig. 1c, top two images).
After 5 years of combined Myfortic ${ }^{\circledR}$ (mycophenolic acid, $720 \mathrm{mg}$ twice per day) and Remicade ${ }^{\circledR}$ (infliximab, $5 \mathrm{mg} / \mathrm{kg}$ every 8 weeks) therapy, BRC fundus lesions did not appear (Fig. 1a, right sextet of 
4 Fig. 1 Patient 6, a male who was 43 years old at diagnosis, with a diagnostic delay of 9 months and a treatment delay of 10 months (right eye). a The left sextet of fundus images shows a fundus at presentation that is slightly fuzzy, but no BRC fundus lesions are visible. The right sextet of images reflects the fundus after 5 years of IST; the fundus has decreased fuzziness and persistent absence of BRC fundus lesions. b The left sextet of FA images was taken at presentation and displays vasculitis of the large vessels, diffuse retinal exudation from the small retinal vessels, cystoid macular oedema, and disc hyperfluorescence. The right sextet of frames reveals almost complete regression of all FA signs after 5 years of IST. c ICGA frames at presentation (top two frames) show numerous HDDs and fuzziness of the choroidal vessels. After 5 years of IST (bottom frames), these typical HDDs have disappeared, but faint areas of hypofluorescence and persistent fuzziness remain visible

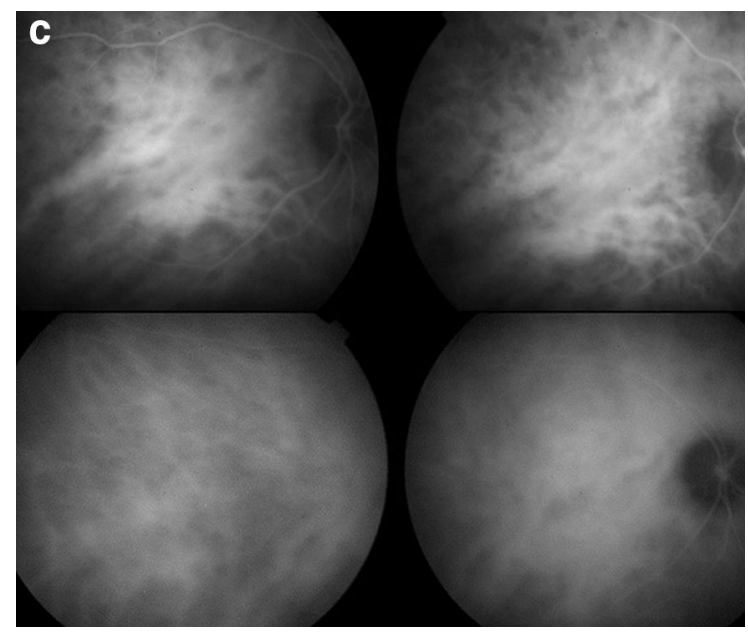

Fig. 1 continued

images). FA results improved markedly, with resolution of vasculitis, macular oedema, and optic disc hyperfluorescence (Fig. 1b, right sextet of images). The ICGA results normalised, with nearly total fading of the HDDs and some persistent fuzziness of vessels, with an angiographic score of 1 (Fig. 1c, bottom images). On follow-up using ICGA frames, it is sometimes difficult to determine whether HDDs are caused by choroidal scars or by active lesions, although the absence of fuzziness of the choroidal vessels suggests that active HDDs are not present [26].

Case 2 (patient 2, early-treatment group, fundus lesions present at entry that resolved, ICGA signs resolved; Fig. 2)

A female patient, aged 54 years, was referred 9 months after the onset of ocular symptomatology consisting of dimness of vision and myodesopsias. She was diagnosed as BRC based on her FA and ICGA findings, her tubular visual fields, and positive HLA-A29 antigen. Despite relatively early diagnosis, fundus examination already revealed typical BRC fundus lesions (Fig. 2a, left sextet of images) associated with the usual FA signs of vasculitis of the large retinal veins, diffuse retinal exudation/leakage from the small capillaries, and disc hyperfluorescence. ICGA showed extensive choroidal involvement, with many HDDs and fuzziness of the choroidal vessels (Fig. 2b, left sextet of images).

IST maintenance therapy consisted of azathioprine $(2.0 \mathrm{mg} / \mathrm{kg})$ and low-dose prednisone after tapering from an initial dose of $1 \mathrm{mg} / \mathrm{kg}$. After 13 years of treatment, not only did the ICGA signs resolve completely (Fig. 2b, right sextet of images), but the BRC fundus lesions resolved as well (Fig. 2a, right sextet of images).

\section{Case 3 (patient 12, late-treatment group, fundus lesions present before treatment that persisted at last follow-up, resolution of ICGA signs; Fig. 3)}

A 61-year-old female patient had been followed elsewhere, but treatment was withheld for 57 months. When she presented, she showed typical bilateral birdshot lesions on fundus examination (Fig. 3a, left sextet of images). FA findings were characterized by massive intraretinal exudation/leakage from the retinal capillaries leading to no staining of the large veins due to the lack of fluorescein concentration in the venous circulation, an angiographic feature often seen in BRC [3]. ICGA indicated a bilateral grade 3 involvement of the choroid, with numerous HDDs (Fig. 3b, left sextet of images).

Treatment, which was decided based on visual field impairment, consisted of dual azathioprine $(2.2 \mathrm{mg} / \mathrm{kg})$ and oral prednisone (40 mg tapered over 4 months). Discrete BRC fundus lesions were initially present in both eyes (Fig. 3a, left sextet of images). 6 years later, the BRC did not show the well-delineated BRC lesions and became substantially fainter although still present (Fig. 3a, right sextet of images). In parallel, the HDDs had completely resolved bilaterally compared to pretreatment (Fig. 3b, right sextet of images). This example of fundus lesions persisting but HDDs resolving indicates that HDDs do not correspond to BRC fundus lesions, which reflect irreversible stromal depigmentation, but usually indicate active lesions. 


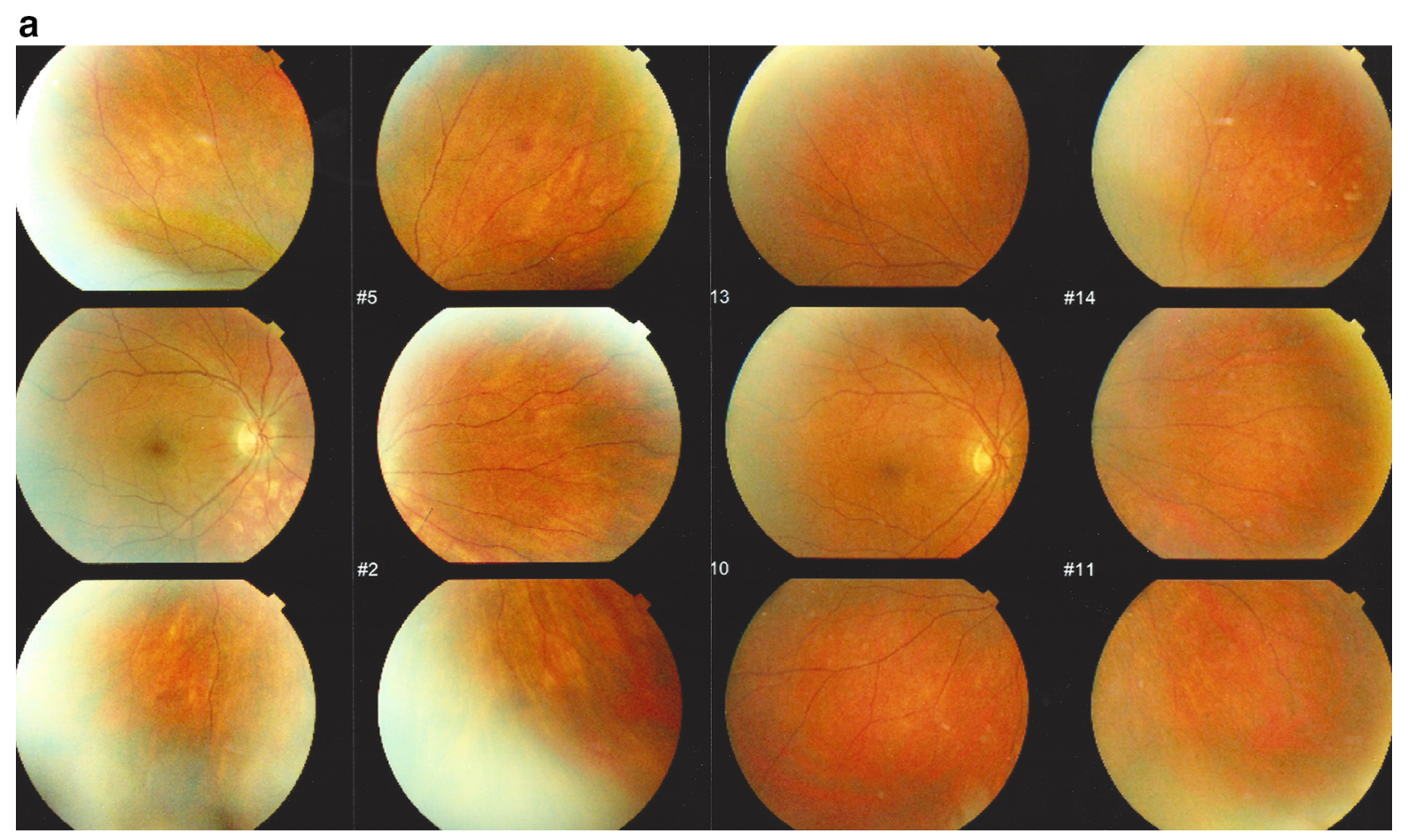

b

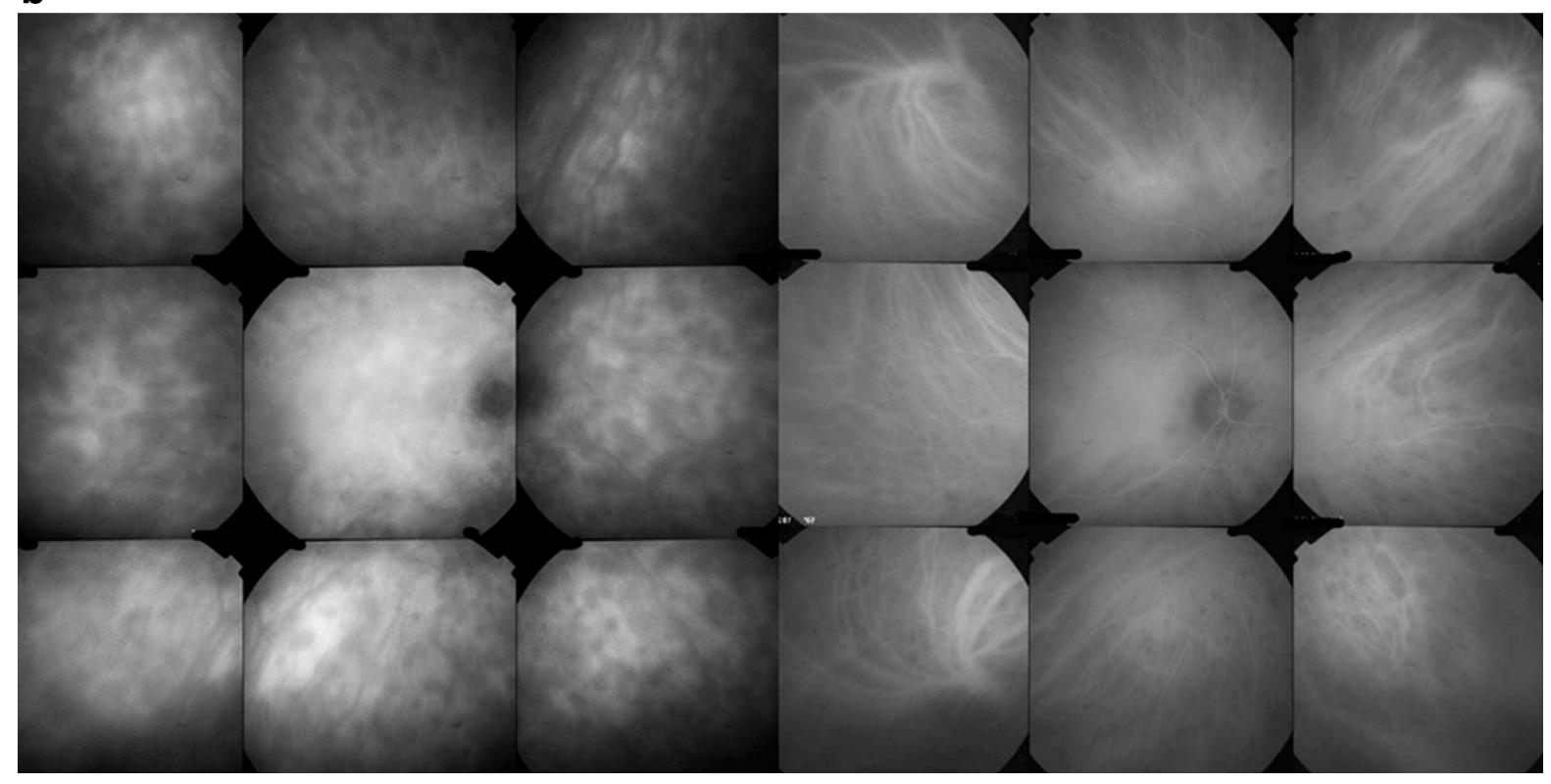

Fig. 2 Patient 2, a female who was 54 years old at diagnosis, with a diagnostic delay of 9 months and a treatment delay of 10 months (right eye). a The left sextet of fundus images shows typical BRC fundus lesions with a fuzzy fundus view. After 13 years of IST, the fundus is less fuzzy and fails to display typical BRC fundus lesions (right sextet of figures). b ICGA frames at presentation (left nine frames) reveal many HDDs with total fuzziness of the choroidal vessels, which cannot be distinguished. The right nine frames indicate an ICGA appearance within normal limits, with resolution of the HDDs and a normal aspect of the large choroidal vessels 


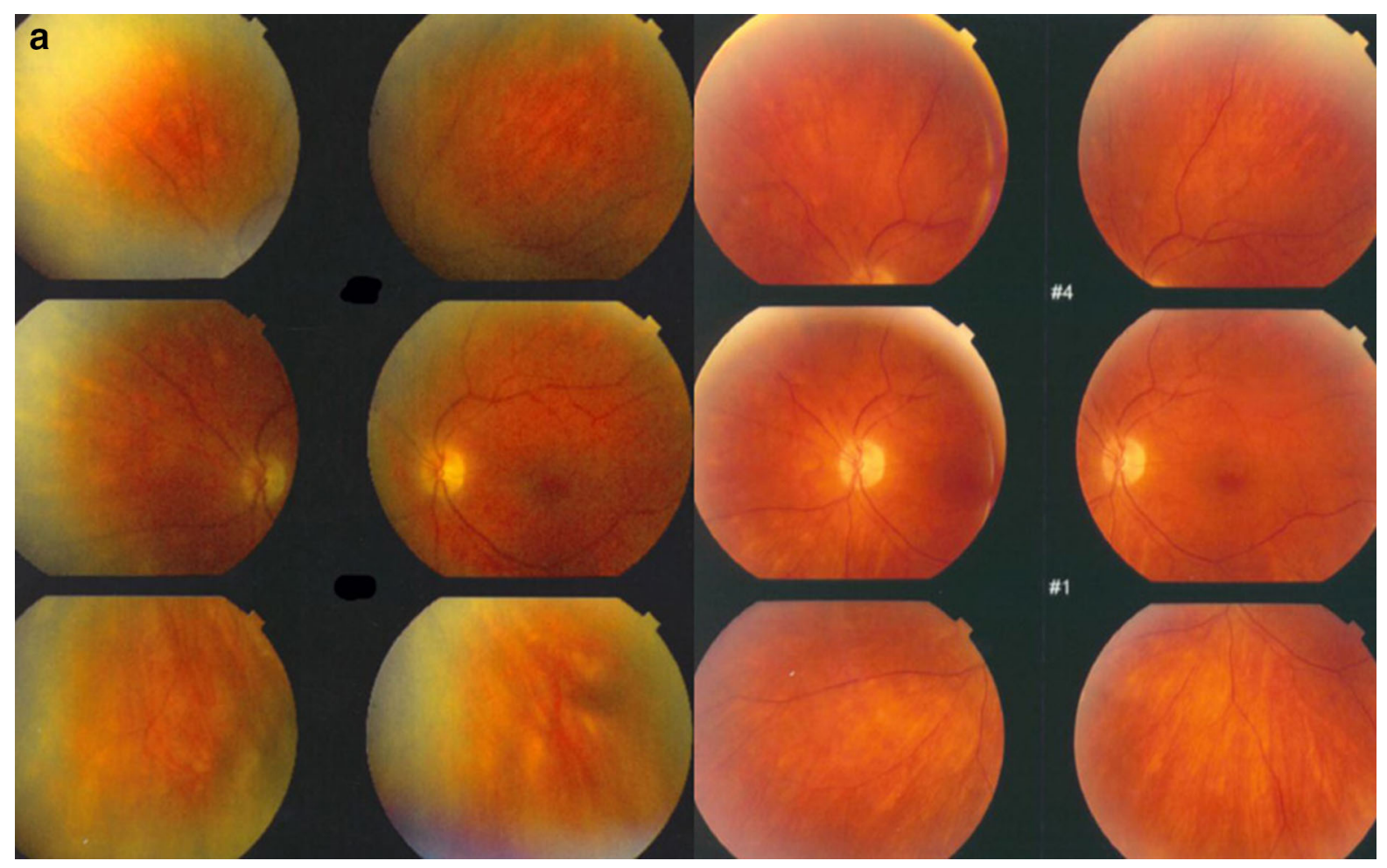

Fig. 3 Patient 12, a female who was 61 years old at presentation, with a diagnostic delay of 6 months and a treatment delay of 57 months (left eye). a The left sextet of images reveals typical, although faint, BRC fundus lesions at presentation that

\section{Discussion}

Since the first descriptions by Ryan and Maumenee [1] and by Gass [2], the hallmark sign of BRC has been the characteristic rice-shaped depigmented fundus lesion. Ryan and Maumenee named BRC after these lesions, which 'frequently have the pattern seen with birdshot in the scatter of a shotgun'. These authors, and subsequently others, described a striking clinical sign of an already-advanced stage of a disease that had not been treated adequately or had not been treated at all because the disease could not be diagnosed at the preBRC fundus lesion stage. This sign served as the basis of BRC diagnosis for the next 33 years.

The peculiar characteristic of BRC is the dual, parallel, but unrelated inflammation of the retina and the choroid [10]. Retinal involvement is best illustrated by FA, which in the active exudative stage of the disease shows diffuse capillary leakage causing diffuse retinal oedema with prominent diffuse hyperfluorescence, sheathing of the larger veins, and tended to become less visible after 6 years of IST (right sextet of fundus images). b ICGA frames at presentation (top six frames) show numerous HDDs that completely resolved after 6 years of IST (bottom six frames)

posterior pole oedema, sometimes with cystoid macular oedema and disc hyperfluorescence [11]. The corollary to this oedematous retinitis is the retinal thickening seen on optical coherence tomography during the exudative phase of the disease [27]. Choroidal inflammation is characterised by a stromal choroiditis that precedes the classical BRC fundus lesions by months or even years, but remains undetected unless ICGA is performed.

We previously reported that ICGA allows the detection of occult choroiditis and, together with compatible retinal findings and the presence of HLAA29 antigen, leads to early diagnosis of BRC before typical fundus lesions are present [28, 29]. If the RCBCR had been applied, patients without the typical BRC fundus lesions would not have been included in the present investigation.

In this study, we compared the evolution of fundus appearance and ICGA signs in two groups of BRC patients receiving IST consisting mostly of $\geq 2$ immunosuppressive/biological agents. Treatment did 


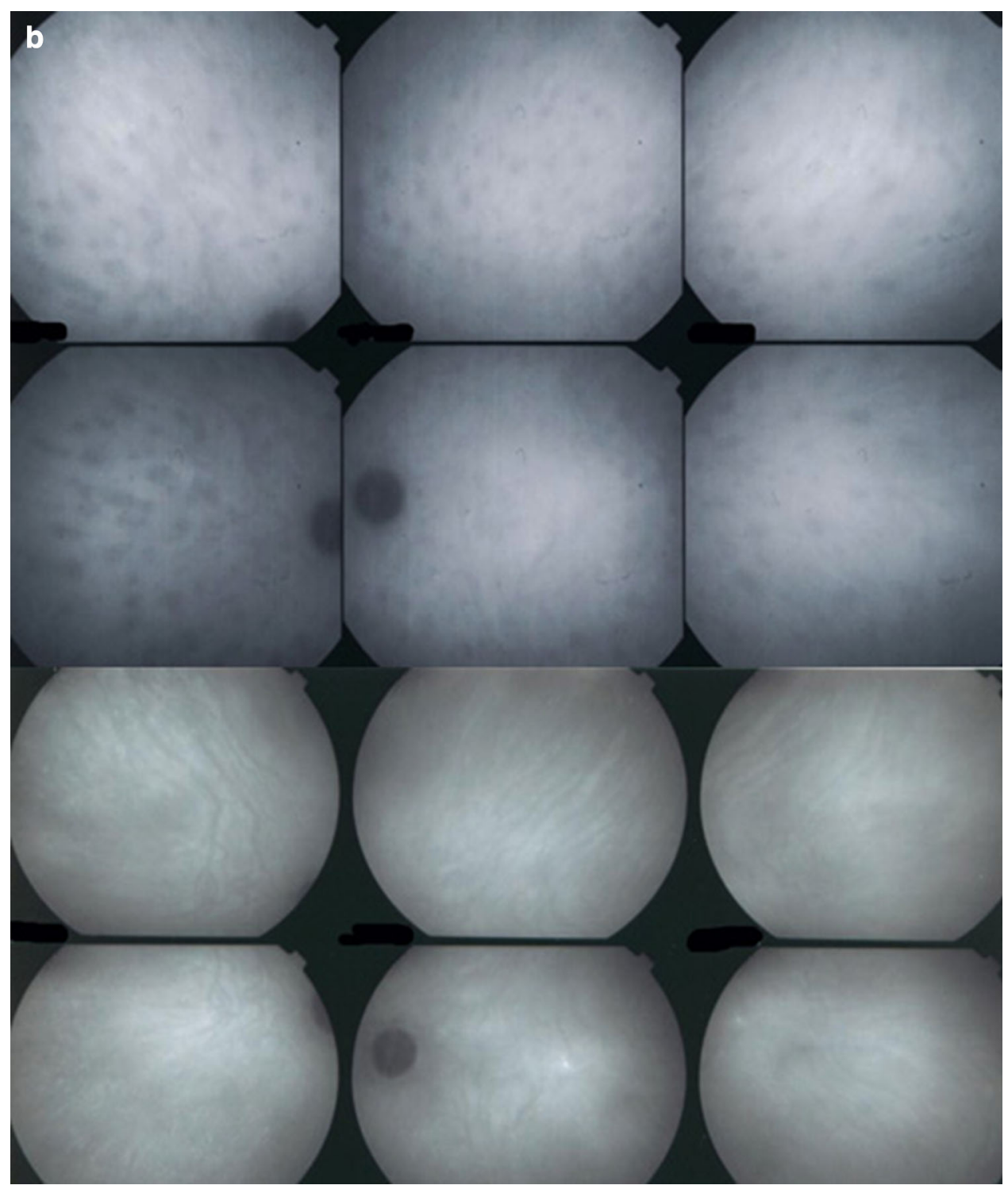

Fig. 3 continued

not differ between groups. In one group, treatment was started early, before the occurrence of BRC fundus lesions. In the other group, mostly due to delays in diagnosis, treatment was started after BRC fundus lesions were already present. We were especially interested in the evolution of the fundus in patients treated before the observation of fundus lesions.

To the best of our knowledge, this is the first report showing that early treatment of BRC nearly completely prevents the development of BRC fundus lesions and modifies the clinical phenotype of the disease. Stromal hypopigmentation of the choroid in these patients did not develop throughout the course of a follow-up of $>10$ years. BRC fundus lesions therefore should be considered to be an evolutionary stage, a complication of the disease when treatment is given too late or is insufficient, but their apparition should not be considered obligatory.

'Regression of birdshot lesions' was previously reported by Leder et al. [30], who in fact described regression of ICGA lesions after treatment was initiated; they treated ICGA lesions and BRC fundus 
lesions as equivalent, which cannot be done because HDDs on ICGA do not correspond to BRC fundus lesions. Our data show that ICGA signs diminished in all of our patients, to almost no signs in the earlytreatment group and to very low values in the latetreatment group, despite the persistence of BRC fundus lesions in the latter group. This observation means that HDDs correspond to active inflammatory lesions that have not yet produced depigmentation and that are still reversible without causing depigmentation. Such lesions were described histologically by Gaudio et al. [31], who indicated that they do not touch the choriocapillaris-retinal pigment epithelium complex. For BRC fundus lesions, upon digestion of the stromal pigment islets, a depigmented area is hypothesised to be left behind that consists of areas that do not produce ICGA signs, because their resolution did not result in significant fibrosis or scarring. At an early stage of the disease, when no BRC fundus lesions are detected, treatment prevents this development, as shown in our study. This evolution is analogous to adequately treated VKH disease. We showed that SGF (which can be considered analogous to BRC fundus lesions) can be prevented by early, sufficiently dosed, and prolonged IST. VKH disease, however, is distinct from BRC because the active and primary inflammation process is limited to the choroid; retinal inflammation is only secondary to the severe choroidal inflammation spilling over to the retina and other neighbouring structures, and successful treatment of choroiditis will also eliminate inflammation in the other compartments. In contrast, control over choroiditis is easily achieved in BRC, as demonstrated by the significant reduction in ICGA score in the two groups of the present investigation. However, this control is not sufficient, as there is autonomous retinal disease that is not secondary to choroiditis $[32,33]$.

In conclusion, we have demonstrated for the first time that early and sufficiently dosed IST can prevent the appearance of typical BRC fundus lesions. It is therefore crucial to perform ICGA to detect otherwise occult stromal choroiditis in suspected BRC cases and to initiate adequate therapy immediately if there is functional impairment, such as visual field changes. The validity of the RCBRC system must be seriously questioned; in addition to its inadequacy for early diagnosis, we recently demonstrated the presence of granulomatous keratic precipitates in 3/19 BRC patients, indicating that this exclusion criterion was inadequate [34]. If we wish to improve outcomes in BRC, diagnostic criteria need to be revised and the main disease-defining criterion of depigmented BRC fundus lesions must be abandoned for the diagnosis of incipient early disease. Waiting for this sign to appear, which is currently necessary for diagnosis, leads to unwanted delay of treatment.

\section{References}

1. Ryan SJ, Maumenee AE (1980) Birdshot retinochoroidopathy. Am J Ophthalmol 89:31-45

2. Gass JDM (1981) Vitiliginous chorioretinitis. Arch Ophthalmol 99:1778-1787

3. Guex-Crosier Y, Herbort CP (1997) Prolonged retinal arterio-venous circulation time by fluorescein but not by indocyanine green angiography in birdshot chorioretinopathy. Ocul Immunol Inflamm 5:203-206

4. Gasch AT, Smith JA, Whitcup SM (1999) Birdshot retinochoroidopathy. Br J Ophthalmol 83:241-249

5. Nussenblatt RB, Mittal KK, Ryan S, Green WR, Maumenee AE (1982) Birdshot retinochoroidopathy associated with HLA-A29 antigen and immune responsiveness to retinal S-antigen. Am J Ophthalmol 94:147-158

6. Baarsma GS, Priem HA, Kijlstra A (1990) Association of birdshot retinochoroidopathy and HLA-A29 antigen. Curr Eye Res 9(Suppl):63-68

7. LeHoang P, Osdemir N, Benhamou A, Tabary T, Edelson C, Betuel H, Semiglia R, Cohen JH (1992) HLA-A29.2 subtype associated with birdshot retinochoroidopathy. Am J Ophthalmol 113:33-35

8. Brezin AP, Monnet D, Cohen JH, Levinson RD (2011) HLA-A29 and birdshot chorioretinopathy. Ocul Immunol Inflamm 19:397-400

9. Wender JD, Fu AD, Jumper JM, McDonald HR, Johnson RN, Cunningham ET Jr (2008) False-negative antibodybased HLA-A29 typing in two patients with birdshot chorioretinopathy. Br J Ophthalmol 92:1153-1154

10. Herbort CP, Probst K, Cimino L, Tran VT (2004) Differential inflammatory involvement in retina and choroid in birdshot chorioretinopathy. Klin Monbl Augenheilkd 221:351-356

11. Papadia M, Herbort CP (2013) Reappraisal of birdshot retinochoroiditis (BRC): a global approach. Graefe's Arch Clin Exp Ophthalmol 251:861-869

12. Levinson RD, Brezin A, Rothova A, Accorinti M, Holland GN (2006) Research criteria for the diagnosis of birdshot chorioretinopathy: results of an international concensus conference. Am J Ophthalmol 141:185-187

13. Lim L, Harper A, Guymer R (2006) Choroidal lesions preceding symptom onset in birdshot chrioretinopathy. Arch Ophthalmol 124:1057-1058

14. Priem HA, Oosterhuis JA (1988) Birdshot chorioretinopathy: clinical characteristics and evolution. Br J Ophthalmol 72:646-659

15. The American Academy of Ophthalmology Basic and Clinical Science Course, The Foundation of the American 
Academy of Ophthalmology, San Francisco (Pub.), Volume 9, Intraocular inflammation and uveitis, 2000-2001 Edition, pp 170-171

16. Nussenblatt RB, Wuitcup SM, Palestine AG (1996) Birdshot retinochoroidopathy. Uveitis, fundamentals and clinical practice, 2nd edn. Mosby, St. Louis, pp 325-333

17. Rothova A, Berendschot TTJM, Probst K, van Kooij B, Baarsma GS (2004) Birdshot chorioretinopathy, long-term manifestations and visual prognosis. Ophthalmology 111:954-959

18. Kiss S, Ahmed M, Leiko E, Foster CS (2005) Long-term follow-up of patients with birdshot retinochoroidopathy treated with corticosteroid-sparing systemic immunomodulatory therapy. Ophthalmology 112:1066-1071

19. Cervantes-Castaneda RA, Gonzalez-Gonzalez LA, Cordero-Coma M, Yilmaz T, Foster CS (2013) Combined therapy of cyclosporine A and mycophenolate mofetil for the treatment of birdshot retinochoroidopathy: a 12-month follow-up. Br J Ophthalmol 97:637-643

20. Bouchenaki N, Herbort CP (2011) Indocyanine green angiography guided management of Vogt-Koyanagi-Harada disease. J Ophthalmic Vis Res 6:241-248

21. Bouchenaki N, Herbort CP (2001) The contribution of indocyanine green angiography to the appraisal and management of Vogt-Koyanagi-Harada disease. Ophthalmology 108:54-64

22. Herbort CP, Mantovani A, Bouchenaki N (2007) Indocyanine green angiography in Vogt-Koyanagi-Harada disease: angiographic signs and utility in patient follow-up. Int Ophthalmol 27:173-182

23. Bacsal K, Wen DS, Chee SP (2008) Concomitant choroidal inflammation during anterior segment recurrence in VogtKoyanagi-Harada disease. Am J Ophthalmol 145:480-486

24. Damico FM, Bezerra FT, Silva GC, Gasparin F, Yamamoto JH (2009) New insights into Vogt-Koyanagi-Harada disease. Arq Bras Oftalmol 72:413-420
25. da Silva FT, Hirata CE, Sakata VM, Olivalves E, Preti R, Pimentel SL, Gomes A, Takahashi WY, Costa RA, Yamamoto JH (2012) Indocyanine green angiography findings in patients with long-standing Vogt-Koyanagi-Harada disease: a cross-sectional study. BMC Ophthalmol 13(12):40

26. Knecht PB, Mantovani A, Herbort CP (2013) Indocyanine green angiography-guided management of Vogt-KoyanagiHarada disease: differentiation between choroidal scars and active lesions. Int Ophthalmol 33(5):571-577

27. Papadia M, Jeannin B, Herbort CP (2012) OCT findings in birdshot chorioretinitis: a glimpse into retinal disease evolution. Ophthalmic Surg Lasers Imaging 43-suppl:S25-S31

28. Fardeau C, Herbort CP, Kullmann N, Quentel G, LeHoang P (1999) Indocyanine green angiography in birdshot chorioretinopathy. Ophthalmology 106:1928-1934

29. Papadia M, Herbort CP (2012) Indocyanine green angiography (ICGA) is essential for the early diagnosis of birdshot chorioretinopathy. Klin Monbl Augenheilkd 229:348-352

30. Leder HA, Galor A, Thorne JE, Jabs D (2008) Disappearance of classic birdshot spots after immunosuppression with tacrolimus and mycophenolate mofetyl. Br J Ophthalmol 92:291

31. Gaudio PA, Kaye DB, Crawford JB (2002) Histopathology of birdshot retinochoroidopathy. $\mathrm{Br} \quad \mathrm{J}$ Ophthalmol 86:1439-1441

32. Hirose T, Katsumi O, Pruett RC, Sakaue H, Mehta M (1991) Retinal function in birdshot retinochroidopathy. Acta Ophthalmol 69:327-337

33. Oh KT, Christmas NJ, Folk JC (2002) Birdshot retinochoroiditis: long-term follow-up of a chronically progressive disease. Am J Ophthalmol 133:622-629

34. Knecht PB, Papadia M, Herbort CP (2013) Granulomatous keratic precipitates in birdshot retinochoroiditis. Int Ophthalmol 33:133-137 